International Journal of Advanced Trends in Computer Science and Engineering

Available Online at http://www.warse.org/IJATCSE/static/pdf/file/ijatcse343942020.pdf

https://doi.org/10.30534/ijatcse/2020/343942020

\title{
Numerical simulation of two aquarius Horizons
}

\author{
N Ravshanov ${ }^{1}$, Sh.K. Daliev ${ }^{2}$, O. Tagaev ${ }^{3}$ \\ ${ }^{1}$ Tashkent University of Information Technologies, 108, Amir Temur str., Tashkent, 100200, Uzbekistan \\ ${ }^{2}$ Samarkand State University, 15, University Boulevard, Samarkand, 140104, Uzbekistan, daliyev.sherzod@mail.ru \\ ${ }^{3}$ Samarkand Economy and Service Institute, Samarkand city, Amir Temur street 9
}

\begin{abstract}
In this study, a mathematical model of groundwater uptake, water accumulation and salt filtration was developed on a two-layer water layer. Two-level reservoirs compiled and numbered a mathematical model of the problem of groundwater recovery. The amount of research is of great practical importance, and in some regions it is necessary to restore saline soils and create clean water resources as an environmentally unfavorable situation.
\end{abstract}

Key words: Groundwater abstraction, salt transfer, mathematical model of filtration, desalination technological schemes, geofiltration process, highly mineralized groundwater.

\section{INTRODUCTION}

Changes in the salinity and chemical composition of groundwater, irrigated areas are determined by hydrochemical, geological, morphological and irrigation conditions, through the mass transfer of soluble substances: convective movement of salts by the filtration stream, diffusion, melting or solidification of salts, and gravitational forces. It affects temperature, pressure gradients, concentration components, the structural state of aqueous solutions and other physicochemical phenomena. Designing powerful and powerful tanks, water intake structures, drainage channels of large gas and oil fields, etc. It has the greatest economic efficiency and requires scientifically based effective methods for solving such problems, which is one of the important problems of applied mathematics. To combat drowning, salinization, flooding, large investments in land reclamation work are required. Therefore, the development of effective ways to solve the problem of predicting changes in groundwater levels is one of the urgent problems.

Recently, hydrogeological modeling has been successfully developing artificial reproduction on various models of groundwater filtration processes and related phenomena to solve hydrogeological problems. It is used not only for a quantitative assessment of filtration conditions in a complex natural environment, but also for a deeper study of the general regional laws of the formation, distribution and movement of groundwater, as well as the scientific justification of the methods and volumes of the designed hydrogeological studies.
Numerical solutions in this case become not only a method of obtaining quantitative characteristics, but also a method of establishing the laws of the process under study. So, on the basis of one or another physical model covering the main aspects of the process, one can obtain the corresponding mathematical model in the form of a certain system of equations that are solved numerically using a personal computers(PC).

The success of the numerical solution of a specific problem is impossible without a clear physical and mathematical formulation, without knowledge of the parameters and initial data of the problem, including the degree of their reliability.

In the numerical solution method, all available methods of studying the problem (analytical tools) should be involved: exact and self-similar solutions obtained under special assumptions, asymptotic estimates, dimensional analysis, and also experimental facts.

As a result of the numerical solution, it is possible to reveal the essential laws of the process, to study the influence of various parameters on it, etc. Then you can move on to other, more complex, physical models. The numerical methods that are implemented on a PC must be economical, that is, spend the minimum number of arithmetic operations; universal, i.e. suitable for a wide class of applied tasks, and simply implemented on a PC. Naturally, the range of such properties includes the convergence and stability of the numerical method.

In [1], hydrodynamic and hydraulic models of water runoff in wetlands are proposed, which allow one to describe the processes of filtration and surface runoff with varying degrees of detail and accuracy. Based on the models of salt transfer by interacting filtration and channel flows, issues of modeling the quality of groundwater and surface water are considered.

In article [2], the concept of groundwater filtration is considered. The types and methods of modeling filtration are described. Particular attention is paid to numerical modeling. Particular attention is paid to numerical modeling. The article discusses the role of computer modeling in hydrogeological research. In addition, the urgency of developing new methods of computer modeling and the creation of a modern information modeling system is emphasized.

In article [3], the process of soaking the aeration zone under various conditions of water supply to the earth's surface is considered. With a power-law dependence of the moisture transfer coefficient and a linear dependence of the suction height on humidity, an analytical function of 
the initial moisture distribution near the groundwater level is obtained. Based on the numerical solution of the corresponding initial-regional problem, the wetting process is analyzed under conditions of close occurrence of groundwater.

In [4], the infiltration rate in the unsaturated zone is analysed from a probabilistic point of view. It is shown that the empirical formulas of Horton and Kostiakov, without apparent physical basis, are explained in a probabilistic approach. Horton's formula reflects a Markovian process contrary to Kostiakov's formula. This approach made it possible to explain why Kostiakov's formula is more powerful than that of Horton.

In article [5], the concept of groundwater filtration is considered. The types and methods of modeling filtration are described. Particular attention is paid to numerical modeling. Particular attention is paid to numerical modeling. The article discusses the role of computer modeling in hydrogeological research. In addition, the urgency of developing new methods of computer modeling and the creation of a modern information modeling system is emphasized.

In [6], a mathematical model of the object of study was proposed, in which the generalized Richards equation was used to transfer water in unsaturated soils. The resulting mathematical model was integrated by the finite-difference method.

In article [7], the process of soaking the aeration zone under various conditions of water supply to the earth's surface is considered. With a power law dependence of the moisture transfer coefficient and a linear dependence of the suction height on humidity, an analytical function of the initial moisture distribution near the groundwater level is obtained. Based on the numerical solution of the corresponding initial-regional problem, the wetting process is analyzed under conditions of close occurrence of groundwater.

In article [8] discusses the process of using the finite difference method for solving problems of predicting groundwater level changes by mathematical modeling of anisotropic aquifers on an inclined aquifer using deterministic models, which simplifies the mathematical description of the initial conditions and the modeling process itself, and also allows the use of standard mathematical apparatus for solving complex problems in non-stationary filtration.

In [9], a model is proposed that allows one to obtain reliable information about changes in the groundwater level and to justify the intensity of water reclamation of agrolandscapes, optimize agricultural drainage calculations, and adjust water management of agricultural lands. Changes in the system of groundwater - the external environment due to the interaction of two generalized kinetic units - the ground flow and the drained zone - are presented schematically. Schematization of groundwater swelling from the catchment allows you to visually come to the balance equation. A methodology and an example of determining the parameters of a model of changing groundwater levels are given. The agreement of the calculation results and the experiment gives all the bases for the practical use of the model.

In [10], a model of the dynamics of groundwater in a stationary flow was proposed and, guided by the law of Darcy's water movement through porous media, we use it to study a $2 \mathrm{D}$ aquifer with a reservoir of water with a constant slope consisting of homogeneous and isotropic media; a more realistic case of uniform anisotropic soil is also being considered. Taking into account some geophysical parameters, we develop a computational procedure in the finite difference method, which solves the obtained elliptic partial differential equation both in a homogeneous isotropic and a homogeneous anisotropic medium. After calibrating the numerical model, this procedure is used to begin the study of the Ayamonte Huelva aquifer in Spain, a modest analysis of the system is given, and we calculate the average discharge vector and its rms value as the first. predicted approximation of fuchs in this system, giving us a signal about the place of best operation; The long-term goal is to develop a complete computing tool for analyzing groundwater dynamics.

In article [11], is a study of the reaction of groundwater to climate change and human impact on Manukan Island in Malaysia. SEAWAT-2000 was used to model groundwater reactions in the study area. A simulation of six scenarios representing climate change and human pressure revealed changes in hydraulic pressures and chloride concentrations. A decrease in pumping speed and an increase in reload speed can change the negative effects of an overdraft on Manukan Island. In general, a decrease in pumping speed and an increase in recharge speed can restore and protect groundwater resources on Manukan Island. Thus, for groundwater management options on Manukan Island, Scenario 2 can reduce the penetration of sea water into the aquifer and maintain water resources on a long-term basis. Choosing Scenario 6 is the best option during the rainy season. The results of this study provide a framework that can be used on other small islands with a similar hydrogeological state to protect groundwater resources.

In article [12], considers the methodology, the results of the construction and practical application of mathematical models of natural-technogenic multi-layer systems. A technique for constructing joint models of groundwater and surface water is presented, computer technology for constructing such systems is given. A technique for modeling filtration and heat transfer processes for constructing models of hydrothermal systems is considered. The systems of regional models for the conditions of the southeastern part of the West Siberian artesian basin and for the central part of the Novomoskovsk industrial district are presented as examples.

In article [13], presents to calculate a uniform rise in the level of groundwater throughout the city of Kharkov, a mathematical model was developed that takes into account additional infiltration into groundwater, the amount of precipitation that infiltrates into groundwater, transpiration, evaporation and groundwater abstraction. 
The developed approach allows the use of numerical methods of assessment to predict the process of changing the groundwater level in built-up areas, taking into account various natural and technogenic factors.

In article [14], the main approaches to modeling moisture transfer in various irrigation methods are considered. The well-known models of moisture transfer in a porouscapillary medium under the action of stationary and unsteady mass forces are analyzed taking into account the non-linear effects that can be used for subsoil irrigation. In this model, the parameters are the groundwater level, the coefficient of water loss; diameters of capillaries, relative volume of capillaries, filtration rate, total inflow and outflow, water level in capillaries, capillary rise speed in capillaries of a certain diameter, capillary rise height, etc. A solution to the mathematical model of moisture transfer of Novoselsky S.N. in frequent derivatives for drip irrigation. An analysis of the mathematical models considered has shown that each of the irrigation methods requires the use of different approaches to mathematical modeling, reflecting the individual sides of this complex process of moisture transfer. For the considered irrigation methods, various approaches to mathematical modeling are applied, reflecting the physical features of the moisture transfer process during drip irrigation. Aggregation of the described models can increase the accuracy of the mathematical description of the moisture transfer processes as a whole, which will clarify the engineering methodology for calculating irrigation and irrigation norms in order to save natural resources, including energy costs.

In [15], a mathematical model was developed to study the distribution of groundwater pressure and its changes in the area of underground cylindrical structures. Based on the created model, the influence of the thickness of the aquifer, soil porosity, filtration coefficient, viscosity coefficient and piezoelectric conductivity coefficient on the pressure that groundwater exerts on the lower part of an underground structure is investigated. The analysis of the possibility of pushing the structure and the destruction of the foundation under the influence of pressure caused by groundwater. Analytical formulas are obtained for assessing the stresses in the foundation and predicting the possibility of its destruction.

In [16], a mathematical model was developed for predicting groundwater levels in two-layer formations. The authors consider a two-layer medium consisting of two layers: soil (with low conductivity) and water, as a mathematical model of a geofiltration process.

In [17], an urgent problem related to the process of change in underground water level and mineral salt transfer in soils is solved in the paper. The problem is described by a system of partial differential equations and the corresponding initial, internal and boundary conditions of various kinds. To derive a mathematical model of the process under consideration, a detailed review of scientific papers devoted to various aspects and software of the object of study is given. To conduct a comprehensive study of the process of filtration and change in salt regime of groundwater, mathematical models and an effective numerical algorithm are proposed taking into account external sources and evaporation. Since the process is described by a nonlinear system of partial differential equations, it is difficult to obtain an analytical solution. In article[18], further developed the model for determining and choosing rational parameters of welding modes, including welding current, for intersecting round rods used in reinforced concrete building structures. It is shown that the solution to present problem requires computerized support, in particular, using the mathematical package Mathcad. Based on computer modeling, experimental studies, as well as analysis of the process of heating the rods, it was found that the current density in the central part of the contact depends only on the length of the contact between the welded rods, as well as on the length of the contact between the electrodes and the rods. A technique has been developed to determine the compression force between the electrodes in the center of the welding contact, taking into account the required minimum welding current of the crossing round rods. The studies carried out made it possible not only analytically, but by computer modeling (Mathcad and Simufact Welding), to justify the rational parameters of the board temperatures of the materials being welded, in particular, round rods in the welding zone and to ensure the reliability and durability of building reinforced concrete structures. In article[19], provides a calculation of the profile, which, at a given radius of curvature, ensures the required zenith angle of multilateral wells of a horizontally branched section at the required depth, the lengths and projections of other characteristic profile sections, the coordinates of the end points of the branches and the values of the zenith angle and azimuth in them when drilling along a given radius. The relevance of the research lies in the development of recommendations for the use of horizontally branched wells in the construction of oil and gas wells, taking into account the geological features and the influence of technological and technical factors in the penetration with horizontal and multilateral wells. Calculations make it possible to choose a well drilling technology that provides maximum development profitability and a reliable, stable operation of the selected wells without complications.

\section{MAIN PART}

According to the results of the analysis of hydrogeological conditions, the territory in terms of geofiltration should be considered as a vertical two-layered medium consisting of two aquifers (with a relatively close permeability value) separated by a poorly permeable layer.

Then, the movement of watermarks (groundwater) under such conditions is described by a system of partial differential equations:

$$
\left.\begin{array}{l}
\mu \frac{\partial h}{\partial t}=-k_{b} \frac{h-H}{m_{b}}+f-\omega, \\
\mu^{*} \frac{\partial H}{\partial t}=-k \frac{H-h}{m}+\frac{\partial}{\partial x}\left(T \frac{\partial H}{\partial x}\right) .
\end{array}\right\}
$$


where, $h(x, t), H(x, t)$ - groundwater and pressure water levels; $\mu$ - coefficient of water loss or lack of saturation; $\mu^{*}$ - coefficient of elastic water loss; $m_{b}, m$-power of the separating layer; $k_{b}, k$-filtration coefficients of the lower and upper layers. conditions:

System (1) is solved under the following initial

$$
h(x, 0)=h_{0}, \quad H(x, 0)=H_{0} .
$$

Here $h_{0}(x), H_{0}(x)$ - are the initial conditions of groundwater and pressure water levels.

Then from (1) we introduce the notation:

$$
\left.\begin{array}{c}
\frac{\partial}{\partial x}\left(T \frac{\partial H}{\partial x}\right)=0, A=-\frac{k_{b}}{\mu m_{b}}, \\
B=-\frac{k}{\mu^{*} m}, \frac{(f-\omega)}{\mu}=q, \\
\frac{\partial h}{\partial t}=A(h-H)+q, \\
\frac{\partial H}{\partial t}=B(H-h) .
\end{array}\right\}
$$

$q=$ const , we differentiate the 1- equation for $t$ in (3).

$$
\frac{\partial^{2} h}{\partial t^{2}}=A \frac{\partial h}{\partial t}-A \frac{\partial H}{\partial t},
$$

Instead, $\frac{\partial H}{\partial t}$ we put the 2-equation of system (3):

$$
\frac{\partial^{2} h}{\partial t^{2}}=A \frac{\partial h}{\partial t}-A \cdot B(H-h),
$$

And $(H-h)$ instead of using the 1 - equation of system (3), we get

$$
\begin{aligned}
& \frac{\partial^{2} h}{\partial t^{2}}=(A+B) \frac{\partial h}{\partial t}-B q \\
& \frac{\partial^{2} h}{\partial t^{2}}-(A+B) \frac{\partial h}{\partial t}=-B q
\end{aligned}
$$

After the designation $\frac{\partial h}{\partial t}=Y,-(A+B)=K,-B q=M$ equation (7) looks like this:

$$
Y^{\prime}+K Y=M \text {. }
$$

The solution to equation (9) will be sought as follows:

$$
\begin{aligned}
& Y=e^{-\int K d t}\left[\int M e^{\int K d t} d t+c\right]=e^{-K t}\left[\int M e^{K t} d t+c\right]= \\
& =e^{-K t}\left[\frac{M}{K} e^{K t}+c\right]=\frac{M}{K}+c e^{-K t}, \\
& \frac{\partial h}{\partial t}=\frac{M}{K}+c e^{-K t} .
\end{aligned}
$$

As a result of integration (10), we obtain:

$$
h=\frac{M}{K} t-\frac{c}{K} e^{-K t}+c_{1},
$$

$H$ similar::

$$
H=\frac{M}{K} t-\frac{s}{K} e^{-K t}+s_{1} .
$$

We use the initial conditions (2) we obtain the above:

$$
\begin{aligned}
& h(x, t)=\frac{B q}{A+B} t+\left(\frac{A\left(h_{0}(x)-H_{0}(x)\right)+q}{A+B}-\frac{B q}{(A+B)^{2}}\right) e^{(A+B) t}+ \\
& +\frac{B}{A+B} h_{0}(x)+\frac{A}{A+B} H_{0}(x)-\frac{q}{A+B}+\frac{B q}{(A+B)^{2}}, \\
& H(x, t)=\frac{B q}{A+B} t+\left(\frac{B\left(H_{0}(x)-h_{0}(x)\right)}{A+B}-\frac{B q}{(A+B)^{2}}\right) e^{(A+B) t}+ \\
& +\frac{A}{A+B} H_{0}(x)+\frac{B}{A+B} h_{0}(x)+\frac{B q}{(A+B)^{2}} .
\end{aligned}
$$

The accepted filtration conditions give reason to present the mathematical model of salt transfer as follows [3,4]:

$$
\left.\begin{array}{l}
n_{0} \frac{\partial c_{0}}{\partial t}=\frac{\partial}{\partial x}\left(D_{x} \frac{\partial c_{0}}{\partial x}\right)-V_{x} \frac{\partial c_{0}}{\partial x} \\
n_{1} \frac{\partial c_{1}}{\partial t}=\frac{\partial}{\partial x}\left(D_{x}^{*} \frac{\partial c_{1}}{\partial x}\right)-V_{x}^{*} \frac{\partial c_{1}}{\partial x} .
\end{array}\right\}
$$

Initial conditions:

$$
c_{0}(x, 0)=c_{00}(x), \quad c_{1}(x, 0)=c_{10}(x) .
$$

Border conditions:

$$
\begin{aligned}
& \left.\left(V_{x} c_{0}-D_{x} \frac{\partial c_{0}}{\partial x}\right)\right|_{x=L_{x}}=0,\left.\frac{\partial c_{1}}{\partial x}\right|_{x=0}=0, \\
& \left.c_{0}(x, t)\right|_{x=m-0}=\left.c_{1}(x, t)\right|_{x=m+0}, \\
& V_{x} c_{0}-\left.D_{x} \frac{\partial c_{0}}{\partial x}\right|_{x=m+0}=V_{x}^{*} c_{1}-\left.D_{x}^{*} \frac{\partial c_{1}}{\partial x}\right|_{x=m-0} .
\end{aligned}
$$

where, $c_{0}(x, t), c_{1}(x, t)$ - is the concentration of salts of soil and pressure aquifers; $V_{x}, V_{x}^{*}\left(V_{x}=-k_{b} \frac{h-H}{m_{b}}\right.$, $\left.V_{x}^{*}=-k \frac{H-h}{m}\right)$ - filtration velocity; $D_{x}, D_{x}^{*}$ - are the diffusion coefficients of salts; $c_{00}(x, t), c_{10}(x, t)$ - the initial distribution of salts in the soil and pressure aquifers; $n_{0}, n_{1}$ - ctive soil porosity in the respective zones.

\section{SOLUTION METHOD}

To solve problem (15) - (19), we use the finite difference method [16,17]. In $D=\left\{0 \leq x<L_{x}, 0 \leq t \leq T\right\}$ the region we introduce a grid, where $T$ is the maximum time during which the process is studied. For this, we divide the interval $[0, a]$, in increments of $\Delta$, and $[0, T]$ in increments. As a result, we have the grid:

$$
\begin{aligned}
& \omega_{h \tau}=\left\{\left(x_{i}, t_{j}\right), i=0,1,2, \ldots N j=0,1, \ldots, J ;\right. \\
& \left.x_{i}=i h ; t_{j}=j \tau ; \tau=T / J, m=h \cdot n\right\}
\end{aligned}
$$

We approximate equation (1) using an explicit scheme on the $\omega_{h \tau}$ grid in the form:

Then from (1) we introduce the notation: $\frac{\partial}{\partial x}\left(T \frac{\partial H}{\partial x}\right)=0$,

$$
\left.\begin{array}{l}
\frac{h_{i}^{j+1}-h_{i}^{j}}{\tau}=-\frac{k_{b}}{\mu \cdot m_{b}}\left(h_{i}^{j}-H_{i}^{j}\right)+\frac{f_{i}^{j}-\omega_{i}^{j}}{\mu} \\
\frac{H_{i}^{j+1}-H_{i}^{j}}{\tau}=-\frac{k}{\mu^{*} \cdot m}\left(H_{i}^{j}-h_{i}^{j}\right)
\end{array}\right\}
$$


Difference scheme (22) is reduced to the system of linear algebraic equations

$$
\left.\begin{array}{l}
h_{i}^{j+1}=\left(1-\frac{\tau_{i} \cdot k_{b}}{\mu \cdot m_{b}}\right) h_{i}^{j}+\frac{\tau_{i} \cdot k_{b}}{\mu \cdot m_{b}} H_{i}^{j}+q_{i} \cdot \tau_{i}, \\
H_{i}^{j+1}=\left(1-\frac{\tau_{i} \cdot k}{\mu^{*} \cdot m}\right) H_{i}^{j}+\frac{\tau_{i} \cdot k}{\mu^{*} \cdot m} h_{i}^{j} .
\end{array}\right\}
$$

The approximation of the initial conditions (2) is defined as follows:

$$
\begin{aligned}
& h_{i}^{0}=h_{0}\left(x_{i}\right) \\
& H_{i}^{0}=H_{0}\left(x_{i}\right)
\end{aligned}
$$

Numerical (a) and analytical (b) results of calculations for the following dimensionless parameters are shown in Figures 1 and 2:

$$
\begin{aligned}
& q=1, \quad \mu=0.006 \quad \mu^{*}=0.003, \quad k_{b}=10.8 \quad k=5.9, \\
& m=4, \quad m_{b}=4.5, \quad x=1 . .10, \quad t=1 . .15
\end{aligned}
$$

These results show that with increasing water depth, the saturation of the soil increases in the form of a square root, and over time this phenomenon changes little.

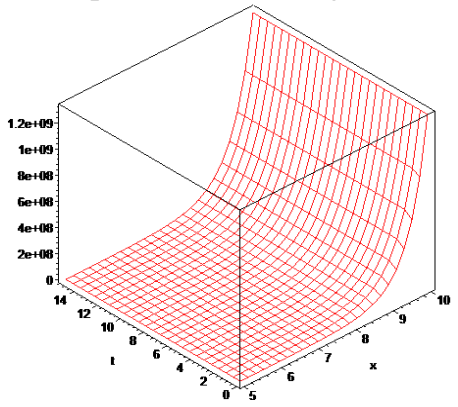

(a) Figure 1: Numerical Results of Calculations

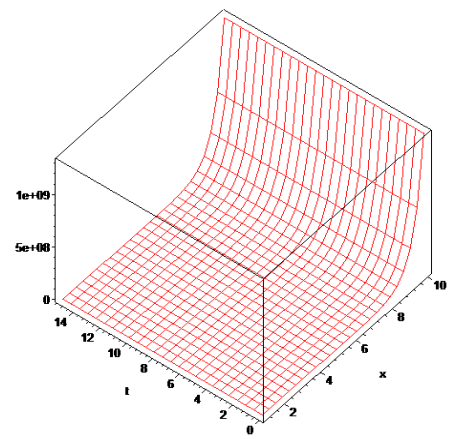

(b) Figure 2: Analytical Results of Calculation

We approximate equation (15), using the implicit scheme $\omega_{h \tau}$ on the grid in the form:

$$
\left.\begin{array}{l}
n_{0} \frac{C_{o i}^{j+1}-C_{o i}^{j}}{\tau}=D_{x} \frac{C_{o i-1}^{j+1}-2 C_{o i}^{j+1}+C_{o i+1}^{j+1}}{\Delta^{2}}-V_{x} \frac{C_{o i}^{j+1}-C_{o i-1}^{j+1}}{\Delta}, \\
n_{1} \frac{C_{1 i}^{j+1}-C_{1 i}^{j}}{\tau}=D_{x}^{*} \frac{C_{1 i-1}^{j+1}-2 C_{1 i}^{j+1}+C_{1 i+1}^{j+1}}{\Delta^{2}}-V_{x}^{*} \frac{C_{1 i}^{j+1}-C_{1 i-1}^{i+1}}{\Delta} .
\end{array}\right\}
$$

The difference scheme (23), is reduced to a system of linear algebraic equations:

$$
\begin{aligned}
& A_{0} c_{0 i-1}^{j+1}-B_{0} c_{0 i}^{j+1}+E_{0} c_{0 i+1}^{j+1}=-F_{0}, i=\overline{1, I-1}, j=\overline{0, J-1}, \\
& A_{1} c_{1 i-1}^{j+1}-B_{1} c_{1 i}^{j+1}+E_{1} c_{1 i+1}^{j+1}=-F_{1}, i=\overline{1, I-1}, j=\overline{0, J-1} .
\end{aligned}
$$

where

$$
\begin{aligned}
& A_{0}=\left(\frac{D_{x} \tau}{n_{0} \Delta^{2}}+\frac{V_{x} \tau}{n_{0} \Delta}\right), B_{0}=\left(\frac{2 D_{x} \tau}{n_{0} \Delta^{2}}+\frac{V_{x} \tau}{n_{0} \Delta}+1\right), E_{0}=\frac{D_{x} \tau}{n_{0} \Delta^{2}}, \\
& A_{1}=\left(\frac{D_{x}^{*} \tau}{n_{1} \Delta^{2}}+\frac{V_{x}^{*} \tau}{n_{1} \Delta}\right), B_{1}=\left(\frac{2 D_{x}^{*} \tau}{n_{1} \Delta^{2}}+\frac{V_{x}^{*} \tau}{n_{1} \Delta}+1\right), E_{1}=\frac{D_{x}^{*} \tau}{n_{1} \Delta^{2}} .
\end{aligned}
$$

The approximation of the boundary conditions (17), (18), (19) is determined as follows:

$$
\begin{gathered}
c_{0 N}^{j+1}-c_{0 N-1}^{j+1}=0, \\
c_{0 N}^{j+1}=c_{0 N-1}^{j+1}, \\
c_{11}^{j+1}=c_{01}^{j+1}, \\
c_{0 n}^{j+1}-c_{1 n}^{j+1}=0, \\
c_{0 n}^{j+1}=c_{1 n}^{j+1}, \\
V_{z} c_{0 n}^{j+1}-D_{z} \frac{c_{0 n+1}^{j+1}-c_{0 n}^{j+1}}{\Delta}=V_{z}^{*} c_{1 n}^{j+1}-D_{z}^{*} \frac{c_{1 n}^{j+1}-c_{0 n-1}^{j+1}}{\Delta} .
\end{gathered}
$$

The solution of systems of equations (24) as watered as follows:

$$
c_{0 i}^{j+1}=\alpha_{0 i-1} c_{0 i-1}^{j+1}+\beta_{0 i+1} .
$$

if we accept the notation $i \approx i+1$, then

$$
c_{0 i+1}^{j+1}=\alpha_{0 i} c_{0 i}^{j+1}+\beta_{0 i}
$$

from this after comparison:

$$
\begin{gathered}
\alpha_{0 i-1}=\frac{A_{0}}{B_{0}-E_{0} \cdot \alpha_{0 i}}, \\
\beta_{0 i-1}=\frac{F_{0}+E_{0} \cdot \beta_{0 i}}{B_{0}-E_{0} \cdot \alpha_{0 i}}, \quad i=N-1, \ldots, n+1 \\
\alpha_{0 N-1}=1, \beta_{0 N-1}=0 .
\end{gathered}
$$

A solution to system (25) is sought as a search sequence for a solution to system of equations (24):

$$
c_{1 i}^{j+1}=\alpha_{1 i+1} c_{1 i+1}^{j+1}+\beta_{1 i+1} .
$$

accept the notation $i \approx i-1$, then

$$
c_{1 i-1}^{j+1}=\alpha_{1 i} c_{1 i}^{j+1}+\beta_{1 i}
$$

from this after comparison:

$$
\begin{gathered}
\alpha_{1 i+1}=\frac{E_{1}}{B_{1}-A_{1} \cdot \alpha_{1 i}}, \\
\beta_{1 i+1}=\frac{F_{1}+A_{1} \cdot \beta_{1 i}}{B_{1}-A_{1} \cdot \alpha_{1 i}}, i=1, \ldots, n-1 \\
c_{0 n}=c_{1 n} \\
\alpha_{11}=1, \quad \beta_{11}=0 .
\end{gathered}
$$

The numerical results of the calculations for the following dimensionless parameters are shown in Figures 1,2 and 3:

$$
\begin{gathered}
q=0, \mu=0.006, \mu^{*}=0.003, k_{b}=10.8, \\
k=5.9, m=4, m_{b}=4.5, n_{0}=0.1, n_{1}=0.3, \\
D_{z}=1 * \exp -5, D_{z}^{*}=2 * \exp -5 .
\end{gathered}
$$




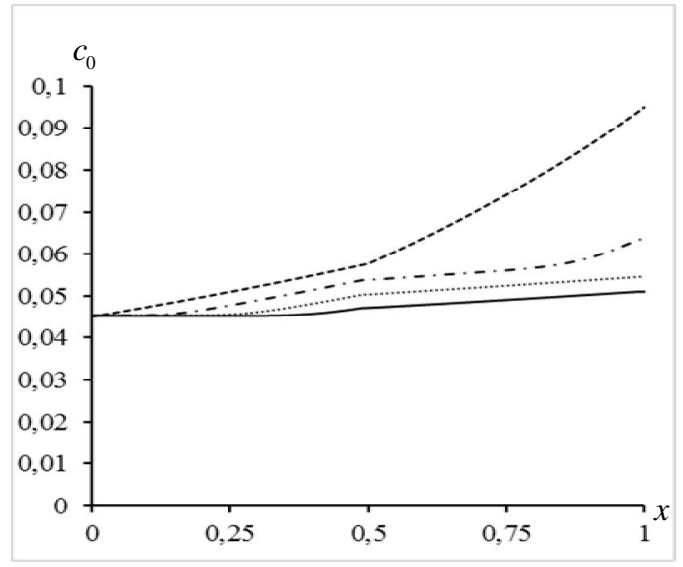

Figure 3: Change profiles

$(---t=8 d a y,-\cdot-t=6$ day, $\cdots t=4$ day,$-t=2$ day $)$

These results show that with increasing depth, the concentration of soil salts increases in the form of a square root, and over time, these phenomena change little

\section{CONCLUSION}

At the same time, the use of a numerical model significantly reduces the volume of field studies, which allows minimizing expensive and material-intensive experimental work, and therefore, reducing the cost of drinking water supplied to consumers.

\section{REFERENCES}

1. Kashevarov A. A. Modeling of water flow and salt transfer processes in wetlands // Applied Mechanics and Technical Physics. - 2005. - No. 1. - P. 96-105.

2. Hantush M. S. Flow to wells in aquiters separated by a semipervious layer. J. Geophys. Res. Vol. 72.No 6. March 15, 1709-1719 P. 1967. - DOI: 10.1029 / JZ072i006p01709

3. P. N. Vabishchevich, A. O. Daniyarov, Mathematical modeling of soaking of the aeration zone in conditions of close occurrence of groundwater, Matem. Mod., 1994, Volume 6, Number 11, P. 11-24.

4. Erick Carlier A probabilistic investigation of infiltration in $\mathrm{t}$ he vadose $\mathrm{z}$ one: proposal for a new $\mathrm{f}$ ormula of infiltration $\mathrm{r}$ ate. Hydrol. Process. 21, P. 2845-2849 (2007) DOI: 10.1002/hyp

5. Troyanskiy M.S. Computer simulation of groundwater filtration. Economics, Statistics and Informatics M: No. 3, 2012. P. 175-178.

6. Pachepsky Y., Timlin D., Rawls W. Generalized Richards 'equation to simulate water transport in unsaturated soils. - Journal of Hydrology, 2003. No. 272. P. 3-13.

7. P.N. Mod., 1994, Volume 6, Number 11, P. 1124.

8. Auzina L.I. Experience using the finite difference method in modeling. Changes in the level of groundwater in the strata on an inclined aquifer. Irgt Bulletin No. 8 (55) 2011.P. 28-33

9. Vasilenkov V.F. , Dyomina O.N. , Aksyonov Y.A. Modeling changes in the level of groundwater with constant water loss.
International Research Journal • No. 4 (46) • Part 2. M. 2016. P. 50-55.

10. V. Vázquez-Báez, A. Rubio-Arellano, D. Garc-aToral, I. Rodr-guez Mora. Modeling an Aquifer: Numerical Solution to the Groundwater Flow Equation. Mathematical Problems in Engineering Volume 2019, Article ID 1613726, 9 pages. doi: 10.1155 / 2019/1613726

11. S. M. Praveena, A. Z. Aris Groundwater resources assessment using numerical model: A case study in low-lying coastal area. Int. J. Environ. Sci. Tech., 7 (1), P.135-146, Winter 2010

12. Krashin I.I., Orfanidi E.K., Semendyaeva L.V., Vishnyakova T.S., Safina T.V., Skvortsova G.A. Numerical modeling of filtration and migration processes to solve hydrogeology problems. exploration and protection of mineral resources. Publisher: All-Russian Scientific Research Institute of Mineral Raw Materials N.M. Fedorovsky. Moscow 2009. P. 33-36.

13. E. A. Strelnikova, E. N. Serikova. Study of the peculiarities of groundwater level changes using mathematical modeling. East European Journal of Advanced Technology ISSN 1729-3774 3/4 (63) 2013.P. 31-35.

14. E.V. Melikhova Mathematical modeling of moisture transfer processes during drip and subsoil irrigation. Lower Volga agricultural university complex: science and higher professional education. News No. 1 (41), 2016. P. 228-234.

15. Valeriy Telichenko, Vladimir Rimshin, Vladimir Kurbatov Mathematical modeling of groundwaters pressure distribution in the underground structures by cylindrical form zone. MATEC Web of Conferences 196, 02025 (2018) https://doi.org/10.1051/matecconf/201819602025

16. Ravshanov N., Daliev Sh. Mathematical model for predicting the groundwater level in two-layer formations // Information technology in modeling and control. - Voronezh: Scientific Book, 2019. No. 2 (116). - P. 116-124.

17. N. Ravshanov, Sh. Daliev 2020 J. Phys.: Conf. Ser. $1441 \quad 012163$. doi:10.1088/17426596/1441/1/012163

18. Ospanova S.M., ShalamberidzeM. Sh., LakhnoV.A., Osypova T. Yu. Mathematical Packages Application in The Process of EnergySaving Technology Development of Metal Structures with Rod Elements Production International Journal of Advanced Trends in Computer Science and Engineering, 9(2), March April 2020, 1375 - 1382. doi.org/10.30534/ijatcse/2020/73922020

19. Nurlan Aldamzharov, Bayan Almatova, Fatyma Balmaganbetova, Dameli Uteulieva, Saltanat Dauletova, Bakutgyl Toksanbaeva Calculation of the Profile of a Horizontally Branched Well. International Journal of Advanced Trends in Computer Science and Engineering, 9(2), March April 2020, 1143 - 1146.

https://doi.org/10.30534/ijatcse/2020/37922020 